\title{
Single-mode photonic crystal nanobeam lasers monolithically grown on Si for dense integration
}

\author{
Taojie Zhou, Mingchu Tang, Member, IEEE, Haochuan Li, Zhan Zhang, Yuzhou Cui, Jae-seong Park, \\ Markel Martin, Thierry Baron, Siming Chen, Senior Member, IEEE, Huiyun Liu, and Zhaoyu Zhang
}

\begin{abstract}
Ultra-compact III-V nanolasers monolithically integrated on $\mathrm{Si}$ with ultra-low energy consumption and small modal volume have been emerged as one of the most promising candidates to achieve $\mathrm{Si}$ on-chip light sources. However, the significant material dissimilarities between III-V and $\mathrm{Si}$ fundamentally limit the performance of Si-based III-V nanolasers. In this work, we report $1.3 \mu \mathrm{m}$ InAs/GaAs quantum-dot photoniccrystal (PhC) nanobeam lasers directly grown on complementary metal-oxide-semiconductor compatible on-axis $\mathrm{Si}(001)$ substrates. The continuous-wave optically pumped $\mathrm{PhC}$ nanobeam lasers exhibited a single-mode operation, with an ultra-low lasing threshold of $\sim 0.8 \mu \mathrm{W}$ at room temperature. In addition, a nanoscale physical volume of $\sim 8 \times 0.53 \times 0.36 \mu \mathrm{m}^{3}\left(\sim 25\left(\lambda n^{-1}\right)^{3}\right)$ was realized through a small number of air-holes in $\mathrm{PhC}$ nanobeam laser. The promising characteristics of the PhC nanobeam lasers with small footprint and ultra-low energy consumption show their advanced potential towards densely integrated Si photonic integrated circuits.
\end{abstract}

Index Terms-Monolithic integration, photonic crystal, nanolasers, quantum dots, silicon photonics.

\section{INTRODUCTION}

The he development of data transmission speed through electronic components has been reaching a bottleneck due to the limited bandwidth and power density, despite new nanofabrication method for advanced microprocessor developed with enormous amount of investment. Accordingly, a new technology with advanced $\mathrm{Si}$ nanophotonics has been emerged as a promising candidate not only for the nextgeneration chip-scale data communication network within data centers, but also for the optical computing which has a higher bandwidth, faster speed, and higher power density than the conventional electronic microprocessor [1-3]. To realize a highly efficient, inter/intra-chip communication on Si platform, a new type of Si-based light-emitting source with ultra-small footprint and ultra-low energy consumption is compulsory.

Recently, monolithic integration of III-V optoelectronic devices on Si platform has fueled impressive developments due to its potential in energy-efficient, low-cost and wafer-scale photonic integrated circuits (PICs), even though wafer bonding technology has been extensively investigated [4]. However, material dissimilarities between III-V materials and $\mathrm{Si}$ including large mismatch in lattice constant, thermal expansion coefficients, as well as polarity, significantly degrade the crystal quality of metamorphic III-V layers on Si $[5,6]$, which result in the degraded device performance. Tremendous research efforts have been made through the optimization of sophisticated epitaxial technologies to realize high-quality epitaxial III-V materials with low defect density on complementary metal-oxide-semiconductor (CMOS)compatible Si or SOI platforms [7-11]. In addition, using III-V quantum dots (QDs) as gain materials for lasers provides various advantages, including insensitivity to defects, low lasing threshold and long lifetime [7, 12]. Owing to the decreased non-radiative recombination ratio, implementing defect-insensitive and high-quality QDs as gain material is regarded as the best solution for highly efficient light-emitting source monolithically grown on Si substrate [13].

Despite considerable research efforts devoted to improving the performance of conventional laser diodes (e.g., ridgewaveguide lasers) grown on Si substrate [13-16], the
Manuscript received July 18, 2021. This work was supported by National Natural Science Foundation of China (No. 62174144), International Cooperation Project (No. 2019A050510002), Shenzhen Key Laboratory Project (No. ZDSYS201603311644527), Shenzhen Fundamental Research Fund (No. JCYJ20150929170644623, No. JCYJ20210324115605016, No. JCYJ20210324120204011), Longgang Key Laboratory Project (No. ZSYS2017003 and No. LGKCZSYS2018000015), Longgang Matching Support Fund (No. CXPTPT-2017-YJ-002 and No. 201617486), Optical Communication Core Chip Research Platform, UK Engineering and Physical Sciences Research Council (EP/P006973/1, EP/T028475/1), National Epitaxy Facility, European project H2020-ICT-PICTURE (780930), Royal Academy of Engineering (RF201617/16/28), French government managed by ANR under the Investissements d'avenir ANR-10-IRT-05 and ANR-15-IDEX-02, French RENATECH network. T Zhou and M. Tang contributed equally to this work.

T. Zhou, M. Tang, J. Park, S. Chen and H. Liu are with the Department of Electronic and Electrical Engineering, University College London, London, Torrington Place, WC1E 7JE, UK (e-mail: mingchu.tang.11@ucl.ac.uk; siming.chen@ucl.ac.uk; huiyun.liu@ucl.ac.uk).

T. Zhou, H. Li, Z, Zhang, Y. Cui and Z. Y. Zhang are with the School of Science and Engineering and Shenzhen Key Lab of Semiconductor Lasers, The Chinese University of Hong Kong, Shenzhen, Guangdong, 518172, P.R. China (email: zhangzy@cuhk.edu.cn).

M. Martin and T. Baron are with Univ. Grenoble Alpes, CNRS, CEA-LETI, MINATEC, Grenoble INP, LTM, F-38054 Grenoble, France. 

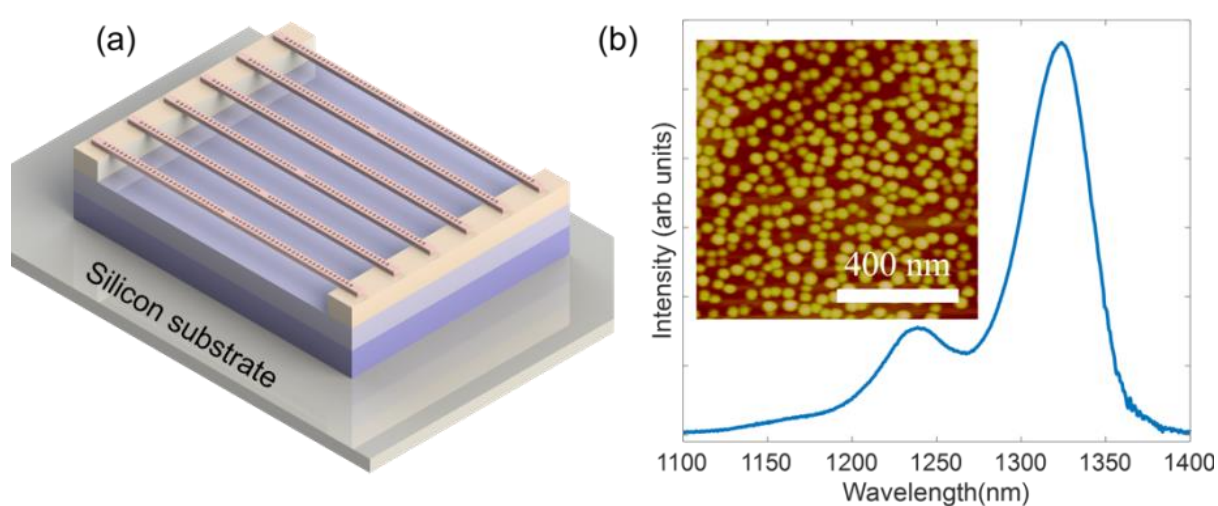

Fig. 1. (a) Schematic diagram of fabricated InAs/GaAs QD PhC nanobeam laser array epitaxially grown on silicon substrate. (b) PL spectra of the as-grown QD gain materials collected at room temperature. The inset shows an AFM image of uncapped InAs/GaAs QDs grown on Si (001) substrate.

integration of nanoscale lasers on CMOS-compatible $\mathrm{Si}$ platforms is still the long-standing goals, which is aiming at densely integrated and more energy-efficient Si-based PICs [17]. Until now, microscale lasers, such as microdisk or microring lasers based on whispering gallery mode [11, 18-20], nano-ridge lasers based on Fabry-Pérot cavity [9, 21], and distributed feedback lasers directly grown on $\mathrm{Si}$ have been developed [22]. All these efforts contributed to laser miniaturization with a range of improvements including in operating temperature and reduction in lasing threshold. In contrast, using photonic crystal $(\mathrm{PhC})$ to construct nanoscale lasers with diffraction-limited modal volume, can provide highly integrated light sources on Si [23, 24]. Moreover, benefiting from its unique properties such as ultra-high quality $(Q)$-factor and enhanced light-matter interaction, ultra-compact $\mathrm{PhC}$ lasers enable promising advantages such as single-mode lasing operation, thresholdless lasing features and high-speed modulation rates [24-28]. Particularly, the simple and robust one-dimensional (1D) waveguide nature of $\mathrm{PhC}$ nanobeam lasers enables a straightforward integration method with other photonic devices [29]. Recently, we presented high performance two-dimensional (2D) QD PhC membrane lasers monolithically grown on CMOS-compatible $\mathrm{Si}$ substrate with a low lasing threshold [24], while the physical size of 2D PhC membrane lasers is $\sim 20 \mu \mathrm{m} \times 20 \mu \mathrm{m}$. In this regard, monolithically integrated nanolasers with more compact size are expected to further increase the integration density, as well as to decrease the lasing threshold.

In this work, we report single-mode, room temperature continuous-wave $(\mathrm{CW})$ optically pumped $1 \mathrm{D} \mathrm{PhC}$ nanobeam lasers monolithically grown on planar CMOS-compatible $\mathrm{Si}$ (001) substrate, of which the footprint approaches as small as $8 \times 0.53 \times 0.36 \mu \mathrm{m}^{3}\left(\sim 25\left(\lambda n^{-1}\right)^{3}\right)$. A tapered air-hole $\mathrm{PhC}$ cavity was implemented to achieve a single high $Q$-factor resonant mode locating within the ground state emission region of as-grown gain material. Single-mode lasing operation with a low lasing threshold of $\sim 0.8 \mu \mathrm{W}$ was obtained for the ultra- compact InAs/GaAs QD PhC nanobeam laser. The ultra-low threshold of PhC nanobeam lasers directly grown on CMOScompatible $\mathrm{Si}$ is comparable with their counterparts on native substrates. The lasing wavelength was also finely tuned over the entire ground state emission region by regularly modifying the structural parameters of devices. The demonstrated Si-based 1D $\mathrm{PhC}$ nanobeam lasers with ultra-small footprint, ultra-low energy consumption and waveguide nature of configuration show their advanced potential towards completely integrated light sources on silicon platform.

\section{MATERIAL EPITAXIAL GROWTH AND DEVICE FABRICATION}

The InAs/GaAs QD PhC nanobeam lasers are epitaxially grown on a CMOS-compatible on-axis $\mathrm{Si}(001)$ substrate, as shown in Fig. 1(a). A $300 \mathrm{~mm}$ diameter Si (001) substrate with a $0.15^{\circ}$ misorientation in the [110] direction was pre-treated under high temperature annealing $\left(900{ }^{\circ} \mathrm{C}\right)$ and hydrogen pressure inside the metal organic chemical vapor deposition system. A $400 \mathrm{~nm}$ of GaAs layer was grown as two steps in order to suppress the formation of antiphase boundaries [30]. The GaAs/Si wafer is then diced into 2-inch wafers for molecular beam epitaxy (MBE) growth. A $200 \mathrm{~nm}$ GaAs buffer layer was regrown in MBE chamber which attributes to a smooth surface examined by a clear reflective high energy electron diffraction pattern. In order to reduce the non-radiative recombination centers caused by high density of threading dislocations due to the large lattice mismatch between GaAs and $\mathrm{Si}$, four sets of defect filter layers (DFLs) were grown, in which each set of DFLs includes five repeats of $\mathrm{In}_{0.18} \mathrm{Ga}_{0.82} \mathrm{As} / \mathrm{GaAs}$ strain-layer superlattices grown at $480{ }^{\circ} \mathrm{C}$ and a $300 \mathrm{~nm}$ GaAs spacing layer grown at $590^{\circ} \mathrm{C}$ [31]. A high temperature in-situ annealing to the strained-layer superlattices were carried out at $590{ }^{\circ} \mathrm{C}$ for 10 minutes. A $1 \mu \mathrm{m} \mathrm{Al} \mathrm{Al}_{0.6} \mathrm{Ga}_{0.4} \mathrm{As}$ sacrificial layer was grown on the top of 


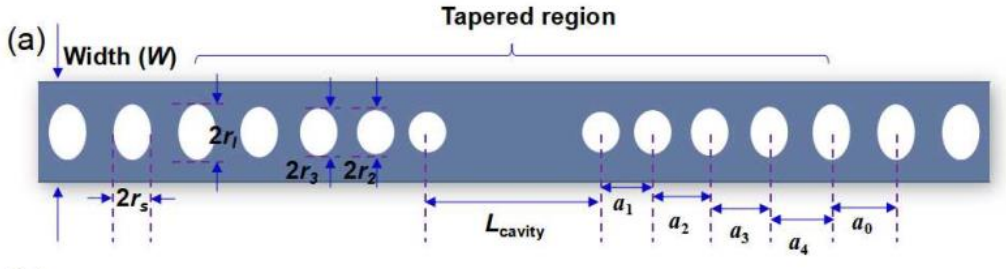

(b)

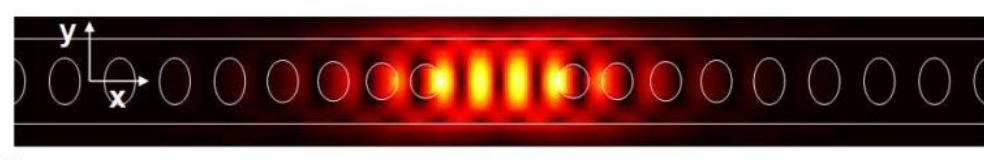

(c)
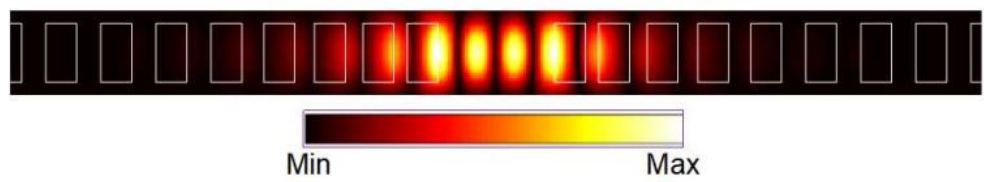

Fig. 2. (a) Top-view schematic of the fabricated InAs/GaAs QD $1 \mathrm{D}$ PhC tapered nanobeam cavity comprised of elliptical air-holes. (b) and (c) present the topview and cross-section view of the calculated E-field profiles for the high $Q$-factor lasing defect mode, calculated by using 3D-FDTD method. The white line represents the boundary of the $\mathrm{PhC}$ nanobeam pattern. (d) TE-like states energy band diagram of the 1D PhC nanobeam structure consisted of periodic elliptical air-holes as depicted in the inset. The structural parameters are $W=524 \mathrm{~nm}, a_{0}=340 \mathrm{~nm}, r_{s} / a_{0}=0.27, r_{l} / r_{s}=1.5$. The shaded green region is the light cone. The violet region indicates the local photonic band gap.

(a)

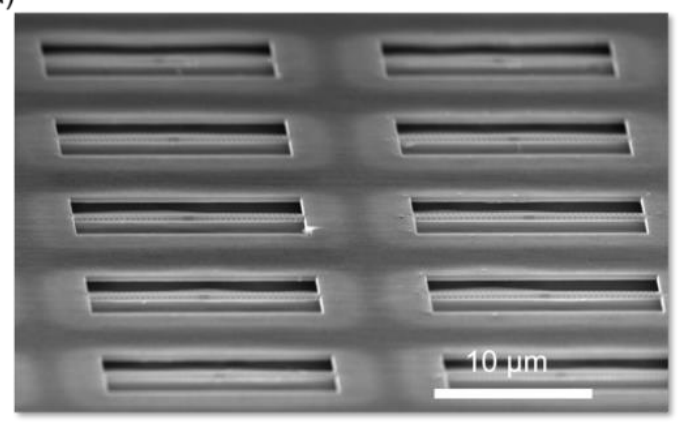

(b)

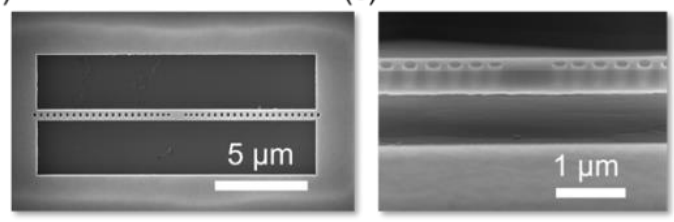

Fig. 3. SEM images of fabricated QD PhC nanobeam laser array (a) and a single nanobeam laser (b) on silicon. (c) A tilted SEM image of tapered cavity region of fabricated $\mathrm{PhC}$ nanobeam laser.

DFLs. Four layers of InAs/GaAs dot-in-well (DWELL) work as active region, which is sandwiched between two thin $40 \mathrm{~nm}$ $\mathrm{Al}_{0.4} \mathrm{Ga}_{0.6} \mathrm{As}$ cladding layers grown at $600{ }^{\circ} \mathrm{C}$. Each layer of DWELL consists of three monolayers of InAs deposited on a 2 $\mathrm{nm} \mathrm{In}_{0.15} \mathrm{Ga}_{0.85}$ As quantum well and capped by a $6 \mathrm{~nm}$ $\mathrm{In}_{0.15} \mathrm{Ga}_{0.85} \mathrm{As}$ layer at $510{ }^{\circ} \mathrm{C}$, which were separated by a $50 \mathrm{~nm}$ high temperature grown $\mathrm{GaAs}$ spacing layer at $590^{\circ} \mathrm{C}$ [32].

A room-temperature micro-photoluminescence ( $\mu$-PL) measurement of the as-grown wafer structure was carried out and the emission spectrum is presented in Fig. 1(b), which indicates a broad ground-state spontaneous emission at around $1.32 \mu \mathrm{m}$ with a narrow linewidth of $\sim 28 \mathrm{meV}$ in O-band. The inset in Fig. 1(b) shows an atomic force microscope (AFM) image of uncapped InAs/GaAs QDs grown on Si (001). The QDs within the active region monolithically grown on $\mathrm{Si}$ substrate present a good uniformity with a density of $\sim 4 \times 10^{10}$ $\mathrm{cm}^{-2}$, and a typical size of $25 \mathrm{~nm}$ in diameter and $8 \mathrm{~nm}$ in height.

A schematic diagram of the fabricated $\mathrm{PhC}$ nanobeam cavity is presented in Fig. 2(a). The $\lambda$-scale cavity region is enclosed between two tapered PhC Bragg mirrors consisting of elliptical air-holes [33, 34], in which the primary lattice constant $\left(a_{0}\right)$ and size of air-holes are linearly tapered down towards smaller value in the cavity region. The structural parameters of the free-standing $\mathrm{PhC}$ nanobeam cavity are $L_{\text {cavity }}$ $=2.64 \times a_{0}, a_{4}=a_{0} \times 0.96, a_{i}=a_{i+1} \times 0.96, r_{i+1} / a_{0}=r_{i} / a_{0}+0.025$ $(i=1-3), r_{l} / r_{s}=1.5$ and a thickness of $\sim 362 \mathrm{~nm}$. To achieve single-mode lasing operation, the $\mathrm{PhC}$ nanobeam cavity was designed by using the three-dimensional finite-difference timedomain (3D-FDTD) method, enabling only a single high $Q$ factor defect mode locating within the ground state emission region of gain materials. Figs. 2(b) and 2(c) depict the top and cross-sectional views of the calculated E-field profiles for the high $Q$-factor resonant defect mode, of which the electromagnetic field is tightly confined by index guiding in $y$ and $z$ directions and Bragg scattering from the periodic $\mathrm{PhC}$ mirror in $x$ direction. The photonic band diagram of designed PhC nanobeam structure was calculated by MIT Photonic Bands (MPB) based on the plane-wave expansion (PWE) method [35]. Fig. 2(d) shows a calculated TE-like states band diagram of the periodic $\mathrm{PhC}$ nanobeam structure with parameters $W=524 \mathrm{~nm}, a_{0}=340 \mathrm{~nm}, r_{s} / a_{0}=0.27, r_{l} / r_{s}=1.5$ and a refractive index $(n)$ of 3.4. The calculated normalized $\mathrm{PhC}$ local optical band gap frequency $\left(a_{0} / \lambda\right)$ extends from 0.208 to 0.278 (from $1223 \mathrm{~nm}$ to $1635 \mathrm{~nm}$ ), 

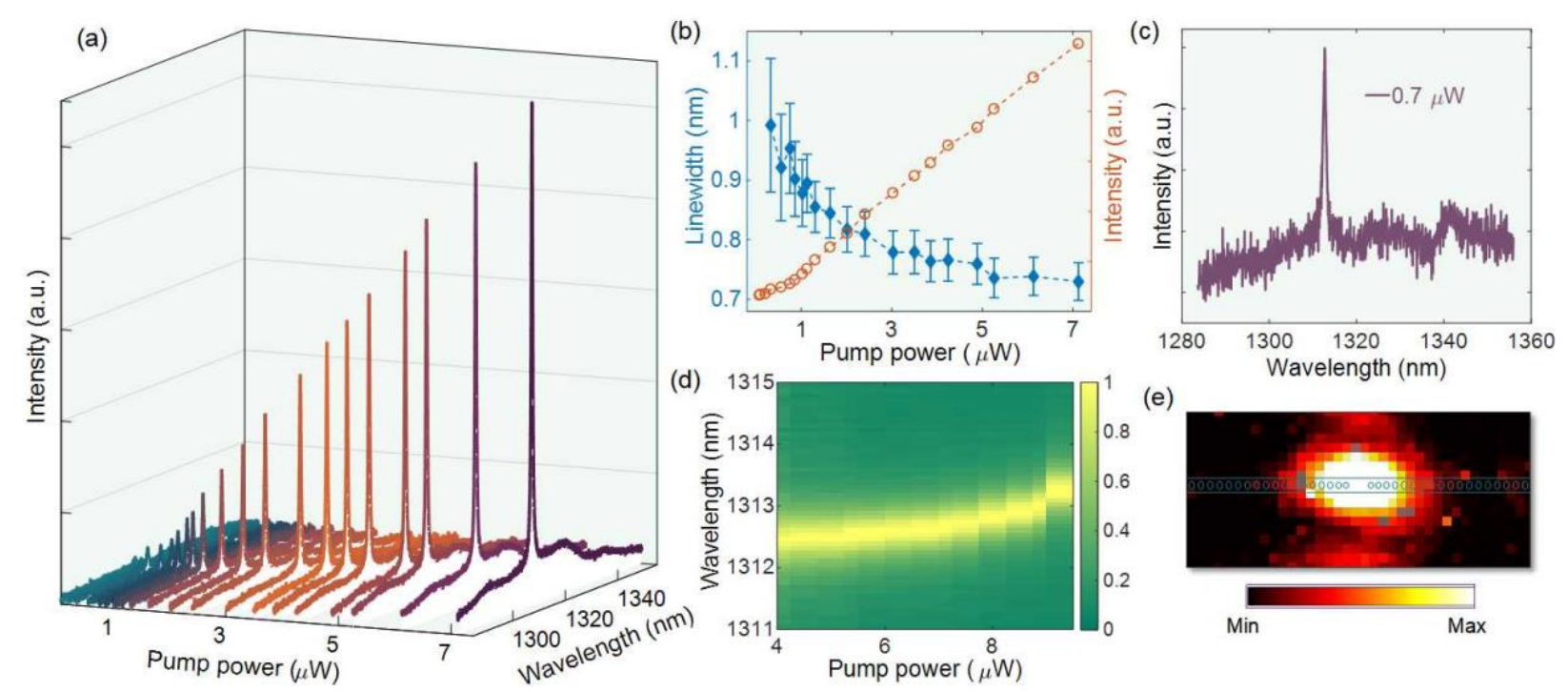

Fig. 4. (a) Power-dependent emission spectra of a PhC nanobeam laser with $W=524 \mathrm{~nm}, a_{0}=340 \mathrm{~nm}, r_{\mathrm{s}} / a_{0}=0.27$. A sharp lasing peak appears above the spontaneous emission background. a.u., arbitrary units. (b) Collected $L-L$ curve and linewidth of the lasing peak at $\sim 1313 \mathrm{~nm}$, indicating a lasing threshold $\sim$ $0.8 \mu \mathrm{W}$. (c) Spectra collected just below the threshold. (d) The lasing wavelength under various input pump powers, presenting a redshift of lasing wavelength due to thermal effect. (e) A mode pattern image taken by an infrared camera. The boundary of the PhC nanobeam laser is also indicated in the image. All experiments were carried out at room temperature.

(a)
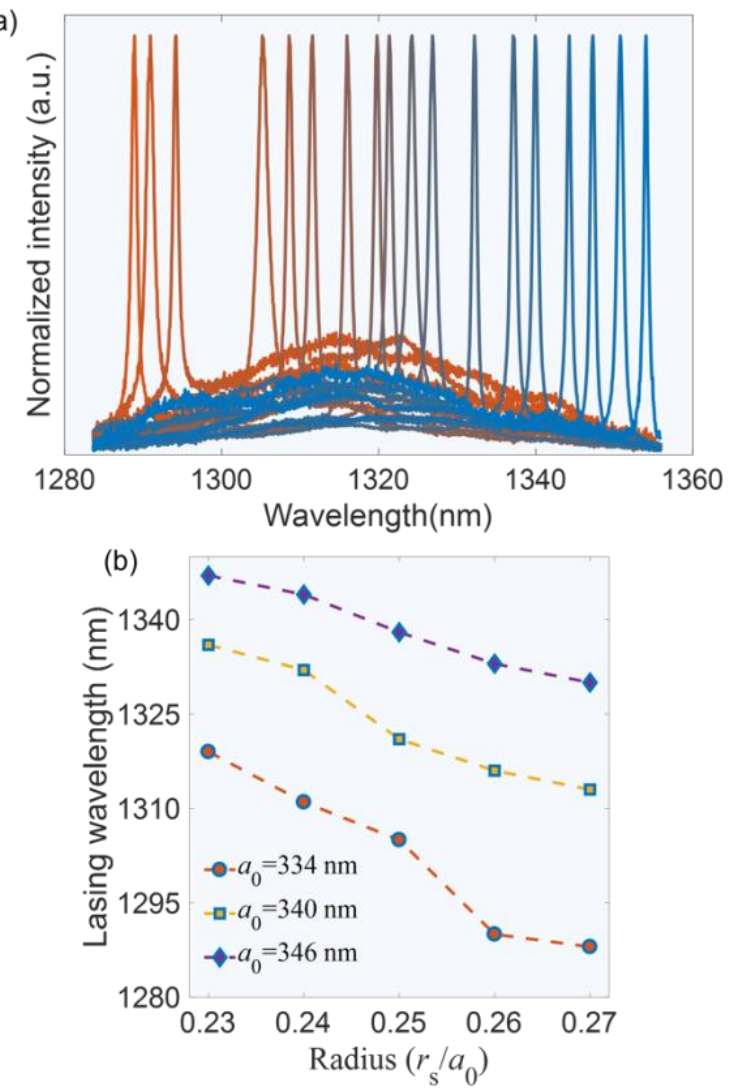

Fig. 5. (a) Normalized measured PL spectra above threshold from representative QD $\mathrm{PhC}$ nanobeam lasers with various structural parameters. (b) The lasing wavelength under various radius $\left(r_{s} / a_{0}\right)$ and lattice constant $\left(a_{0}\right)$ of $\mathrm{PhC}$ nanobeam lasers on silicon.

covering the ground-state emission region of as-grown QDs.

The freestanding 1D PhC nanolasers on $\mathrm{Si}$ were fabricated by the following nanofabrication processes. A layer of $120 \mathrm{~nm}$ silicon dioxide as a hard etching mask was deposited on the asgrown wafer by plasma-enhanced chemical vapor deposition. After the wafer was spin-coated with electron beam resist ZEP$520 \mathrm{~A}$, the $\mathrm{PhC}$ nanobeam pattern was first defined by using electron beam lithography, and then the pattern was transferred into the hard mask by using plasma etching. Afterwards, the electron beam resist was etched by using $\mathrm{O}_{2}$ plasma. Then chlorine-based inductively coupled plasma reactive ion etching (ICP-RIE) was implemented to achieve $\mathrm{PhC}$ nanobeam structure within gain region. Then, wet etching method was subsequently performed to remove the residual silicon oxide and the sacrificial layer to form a suspended $\mathrm{PhC}$ nanobeam.

$\mathrm{PhC}$ nanobeam cavity arrays were fabricated by systematically varying the $a_{0}$ and $r_{s}$ of air-holes, resulting in nanolasers with different operating wavelengths. A SEM image of fabricated nanobeam cavity array is depicted in Fig. 3(a), and Fig. 3(b) presents the SEM image of a single PhC nanobeam cavity with a length ( $x$ direction) of $\sim 15 \mu \mathrm{m}$ and the width (W) of $\sim 524 \mathrm{~nm}$. Vertical and smooth etched air-holes are expected to play a significant role in the performance of $\mathrm{PhC}$ nanobeam lasers, especially to minimize the non-radiative surface recombination effect and achieve $\mathrm{CW}$ pumped nanolasers operated at room temperature. As presented in Fig. 3(c), a magnified view of cavity region indicates smooth profiles of etched air-holes, which contributes to the low lasing threshold of fabricated devices.

\section{NANOLASER OPTICAL MEASUREMENT}

The fabricated nanobeam laser were CW optically pumped using a $632.8 \mathrm{~nm}$ He-Ne laser as excitation light in a surfacenormal $\mu$-PL measurement system. The pumping spot was 
focused by a $50 \times$ objective with a numerical aperture of 0.42 and was carefully positioned in the $\mathrm{PhC}$ nanobeam cavity
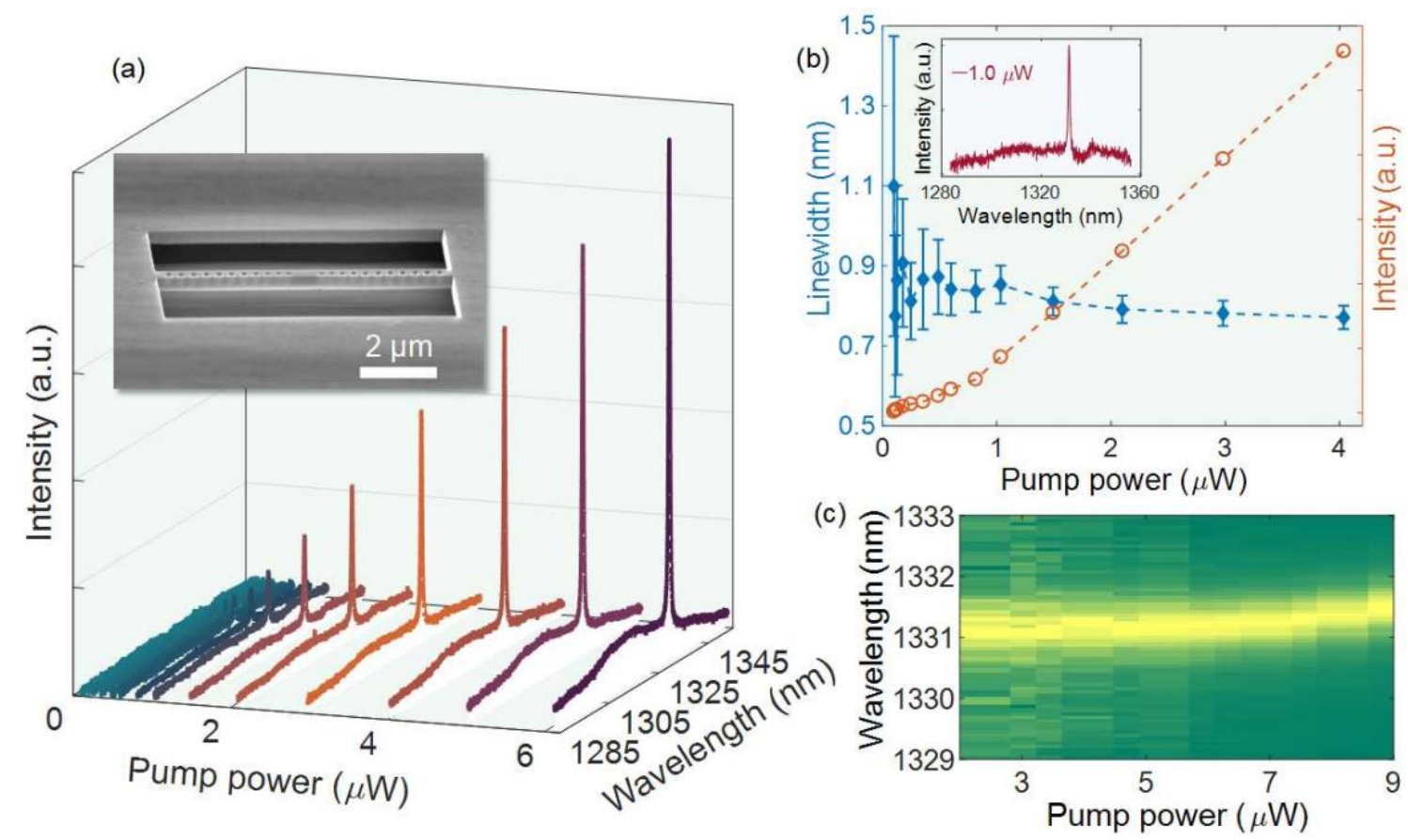

Fig. 6. (a) Power-dependent emission spectra of a more compact single-mode PhC nanobeam laser on silicon. The structural parameters are $W=530 \mathrm{~nm}, a_{0}=$ $346 \mathrm{~nm}, r_{\mathrm{s}} / a_{0}=0.26$. Inset: A tilted SEM image of the fabricated nanobeam laser. (b) Collected $L-L$ curve and linewidth of the lasing peak at $\sim 1331 \mathrm{~nm}$, indicating a lasing threshold $\sim 0.9 \mu \mathrm{W}$. (c) The lasing wavelength under various input pump powers.

region by using piezo-electric nanopositioners. The diameter of the pump spot size is around $1.2 \mu \mathrm{m}$ to $2.5 \mu \mathrm{m}$ under implemented incident pump powers. And the pump powers of the fabricated nanobeam lasers were directly measured by replacing the fabricated devices with a power meter. The output light of PhC nanobeam lasers was collected from the top by using the same objective and analyzed by a monochromator with an InGaAs detector cooled by liquid nitrogen. Singlemode lasing emission was observed for many $\mathrm{PhC}$ nanobeam lasers fabricated on the same wafer. Fig. 4(a) depicts measured power-dependent spectra of a single-mode $\mathrm{PhC}$ nanobeam laser with structural parameters $W=524 \mathrm{~nm}, a_{0}=340 \mathrm{~nm}$ and $r_{s} / a_{0}$ $=0.27$. A sharp lasing peak $(\sim 1313 \mathrm{~nm})$ is observed above the spontaneous emission background, which is within the ground state emission of QDs. Mainly caused by the fluctuations in fabrication, a slight difference exists between the designed lasing wavelength and measured value, as well as the measured $Q$-factor $(Q=\lambda / \Delta \lambda=1500)$ is smaller than the calculated value ( 4000). The collected $L-L$ (light-out versus light-in) curve and the linewidth of the lasing peak under various pump powers are shown in Fig. 4(b), providing the evidence of the lasing operation with a kink of $L-L$ curve and the spectral linewidth narrowing effect. The measured lasing threshold $\left(P_{t h}\right)$ is around $0.8 \mu \mathrm{W}$ driven from the $L-L$ curve, which should be lower if considering the absorption efficiency of excitation light and the effective pump area [36]. The lasing spectra measured just below the threshold with a growing resonant peak from the spontaneous emission background is depicted in Fig. 4(c). The power-dependent measured lasing wavelengths present a clear redshift under increased pump power as displayed in Fig. 4(d), which was mainly induced by the change of the refractive index due to the heating of the cavity. Fig. 4(e) shows a mode pattern image taken above threshold by an infrared camera, indicating a strong light spot in the central cavity region of $\mathrm{PhC}$ nanobeam laser.

The feasibility of tunable nanolasers on $\mathrm{Si}$ were preliminarily tested by varying the structural parameters of designed PhC nanobeam cavities. As illustrated in Fig. 5(a), a wide range of tunable lasing wavelengths around the ground state region was achieved in the fabricated $\mathrm{PhC}$ nanobeam lasers. For nanobeam lasers operating away from the central peak of ground state emission, a relatively intense spontaneous emission background exists in the collected lasing spectra, which is expected to be further suppressed by increasing the layers of QDs and optimizing the etching profiles of devices. The size of air-holes or lattice constant varies slowly across the designed $\mathrm{PhC}$ nanobeam array, resulting in a gradual shift in the lasing wavelength. Though there could be slight discrepancies between measured and designed wavelengths owing to the fabrication fluctuations such as the edge roughness of air-holes, the lasing wavelengths show a regular trend under various radius $\left(r_{s} / a_{0}\right)$ and lattice constant $\left(a_{0}\right)$ as presented in Fig. 5(b). The lasing wavelengths were systematically blue-shifted with increasing the radius of air-holes (or decreasing the lattice constant) due to the decreased effective refractive index. This feature of tunable wavelength may be useful for the future development of ultra-compact dense-wave-divisionmultiplexing light sources on $\mathrm{Si}$. 
To further pursuit compact size of the $\mathrm{PhC}$ laser, $\mathrm{PhC}$ nanobeam cavity with less air-holes was designed to decrease the size of integrated lasers on $\mathrm{Si}$, even though suffering from a weakened mode confinement. However, the proper choice and design of the numbers of air-holes will not degrade the $Q$-factor critically. Therefore, lasing operation can still be sustained within such a small $1 \mathrm{D}$ PhC nanocavity. This kind of compact nanobeam laser with tapered cavity region also has the capability for the future integrated electrically pumped nanolasers on Si [37]. Fig. 6(a) presents power-dependent lasing spectra of a single-mode $\mathrm{PhC}$ nanobeam laser with a small physical volume of $\sim 8 \times 0.53 \times 0.36 \mu \mathrm{m}^{3}\left(\sim 25\left(\lambda n^{-1}\right)^{3}\right)$. The inset of Fig. 6(a) shows a tilted SEM image of the fabricated ultra-compact 1D PhC nanobeam laser. The measured output PL intensity and the linewidth of the lasing peak as a function of incident pump powers are shown in Fig. 6(b), which exhibits the evidence of the lasing operation with a threshold estimated $\sim 0.9 \mu \mathrm{W}$. The inset of Fig. $6(\mathrm{~b})$ shows the measured spectra just above the threshold. A redshift of lasing peak under higher pump power was also observed in this ultracompact 1D PhC nanobeam laser, as presented in Fig. 6(c). The presented lasing threshold $P_{t h}$ of the fabricated 1D $\mathrm{PhC}$ nanolasers directly grown on $\mathrm{Si}$ is in the same order of $\mathrm{PhC}$ lasers grown on its native substrate [38-40], which is mainly attributed to the insensitivity to defects and high crystal quality of the grown QDs on $\mathrm{Si}$. A fabricated high $Q$-factor $\mathrm{PhC}$ nanocavity is also essential to achieve the laser operation at room temperature under $\mathrm{CW}$ excitation [38].

\section{CONCLUSION}

In conclusion, we report ultra-compact InAs/GaAs QD 1D $\mathrm{PhC}$ nanobeam lasers monolithically grown on a planar CMOScompatible $\mathrm{Si}$ (001) substrate. The fabricated $\mathrm{PhC}$ nanobeam lasers were $\mathrm{CW}$ optically pumped under room temperature, exhibiting a single-mode lasing operation and a low lasing threshold of $\sim 0.8 \mu \mathrm{W}$. Besides, multi-integrated laser sources were preliminarily studied by slightly tuning the lattice constant or radius of air-holes of a PhC nanobeam laser array. The demonstrated ultra-compact Si-based 1D PhC nanobeam lasers in this work are expected to play a key role in the photonic applications such as short-distance optical communication and data centers.

\section{ACKNOWLEDGMENT}

The authors would like to thank Xuexuan Qu, Yao Wang and Rui Zhang from the Core Research Facilities of Southern University of Science and Technology for the technical help in device fabrication.

\section{REFERENCES}

R. Soref, "The past, present, and future of silicon photonics," IEEE Journal of selected topics in quantum electronics, vol. 12, no. 6, pp. 1678-1687, 2006

[2] T. Komljenovic, D. Huang, P. Pintus, M. A. Tran, M. L. Davenport, and J. E. Bowers, "Photonic integrated circuits using heterogeneous integration on silicon," Proceedings of the IEEE, vol. 106, no. 12, pp. 2246-2257, 2018

[3] C. Sun et al., "Single-chip microprocessor that communicates directly using light," Nature, vol. 528, no. 7583, pp. 534-538, 2015.

[4] R. Helkey, A. A. Saleh, J. Buckwalter, and J. E. Bowers, "Highperformance photonic integrated circuits on silicon," IEEE Journal of Selected Topics in Quantum Electronics, vol. 25, no. 5, pp. 1-15, 2019.

[5] Q. Li and K. M. Lau, "Epitaxial growth of highly mismatched III-V materials on (001) silicon for electronics and optoelectronics," Progress in Crystal Growth and Characterization of Materials, vol. 63, no. 4, pp. 105-120, 2017.

[6] H. Kroemer, "Polar-on-nonpolar epitaxy," Journal of Crystal Growth, vol. 81, no. 1-4, pp. 193-204, 1987.

[7] S. Chen et al., "Electrically pumped continuous-wave III-V quantum dot lasers on silicon," Nature Photonics, vol. 10, no. 5, pp. 307-311, 2016.

[8] M. R. Calvo et al., "Mid-infrared laser diodes epitaxially grown on on-axis (001) silicon," Optica, vol. 7, no. 4, pp. 263-266, 2020.

[9] Y. Han, Z. Yan, W. K. Ng, Y. Xue, K. S. Wong, and K. M. Lau, "Bufferless $1.5 \mu \mathrm{m}$ III-V lasers grown on Si-photonics $220 \mathrm{~nm}$ silicon-on-insulator platforms," Optica, vol. 7, no. 2, pp. 148-153, 2020.

[10] Z.-H. Wang, W.-Q. Wei, Q. Feng, T. Wang, and J.-J. Zhang, "InAs/GaAs quantum dot single-section mode-locked lasers on $\mathrm{Si}$ (001) with optical self-injection feedback," Optics Express, vol. 29, no. 2, pp. 674-683, 2021/01/18 2021.

[11] W.-Q. Wei et al., "Phosphorus-free $1.5 \mu \mathrm{m}$ InAs quantum-dot microdisk lasers on metamorphic InGaAs/SOI platform," Optics letters, vol. 45, no. 7, pp. 2042-2045, 2020.

[12] J. C. Norman et al., "A review of high-performance quantum dot lasers on silicon," IEEE Journal of Quantum Electronics, vol. 55, no. 2, pp. 1-11, 2019.

[13] M. Tang et al., "Integration of III-V lasers on Si for Si photonics," Progress in Quantum Electronics, vol. 66, pp. 1-18, 2019.

[14] J. Kwoen, B. Jang, K. Watanabe, and Y. Arakawa, "Hightemperature continuous-wave operation of directly grown InAs/GaAs quantum dot lasers on on-axis $\mathrm{Si}$ (001)," Optics express, vol. 27, no. 3, pp. 2681-2688, 2019.

[15] S. Liu et al., "High-channel-count $20 \mathrm{GHz}$ passively mode-locked quantum dot laser directly grown on $\mathrm{Si}$ with $4.1 \mathrm{Tbit} / \mathrm{s}$ transmission capacity," Optica, vol. 6, no. 2, pp. 128-134, 2019.

[16] J. Kwoen, B. Jang, J. Lee, T. Kageyama, K. Watanabe, and Y. Arakawa, "All MBE grown InAs/GaAs quantum dot lasers on onaxis Si (001)," Optics express, vol. 26, no. 9, pp. 11568-11576, 2018.

[17] M. T. Hill and M. C. Gather, "Advances in small lasers," Nature Photonics, vol. 8, no. 12, pp. 908-918, 2014.

[18] Y. Wan et al., "Low-threshold continuous-wave operation of electrically pumped $1.55 \mu \mathrm{m}$ InAs quantum dash microring lasers," ACS Photonics, vol. 6, no. 2, pp. 279-285, 2018.

[19] T. Zhou et al., "Ultra-low threshold InAs/GaAs quantum dot microdisk lasers on planar on-axis $\mathrm{Si}(001)$ substrates," Optica, vol. 6, no. 4, pp. 430-435, 2019/04/20 2019.

[20] S. Zhu, B. Shi, and K. M. Lau, "Electrically pumped $1.5 \mu \mathrm{m} \mathrm{InP-}$ based quantum dot microring lasers directly grown on (001) $\mathrm{Si}$," Optics letters, vol. 44, no. 18, pp. 4566-4569, 2019.

[21] Y. Han, W. K. Ng, Y. Xue, Q. Li, K. S. Wong, and K. M. Lau, "Telecom InP/InGaAs nanolaser array directly grown on (001) silicon-on-insulator," Optics letters, vol. 44, no. 4, pp. 767-770, 2019.

[22] Z. Wang et al., "Room-temperature InP distributed feedback laser array directly grown on silicon," Nature Photonics, vol. 9, no. 12, pp. 837-842, 2015.

[23] S. Mauthe et al., "Hybrid III-V Silicon Photonic Crystal Cavity Emitting at Telecom Wavelengths," Nano Letters, vol. 20, no. 12, pp. $8768-8772,2020$

[24] T. Zhou et al., "Continuous-wave quantum dot photonic crystal lasers grown on on-axis Si (001)," Nature Communications, vol. 11, no. 1, p. 977, 2020/02/20 2020.

[25] Y. Akahane, T. Asano, B.-S. Song, and S. Noda, "High-Q photonic nanocavity in a two-dimensional photonic crystal," Nature, vol. 425, no. 6961, pp. 944-947, 2003/10/01 2003.

[26] S. Matsuo et al., "High-speed ultracompact buried heterostructure photonic-crystal laser with $13 \mathrm{fJ}$ of energy consumed per bit 
transmitted," Nature Photonics, vol. 4, no. 9, pp. 648-654, 2010/09/01 2010.

[27] B. Ellis et al., "Ultralow-threshold electrically pumped quantum-dot photonic-crystal nanocavity laser," Nature Photonics, vol. 5, no. 5, pp. 297-300, 2011/05/01 2011.

[28] I. Prieto et al., "Near thresholdless laser operation at room temperature," Optica, vol. 2, no. 1, pp. 66-69, 2015/01/20 2015.

[29] Y. Halioua et al., "Hybrid III-V semiconductor/silicon nanolaser," Optics Express, vol. 19, no. 10, pp. 9221-9231, 2011/05/09 2011.

[30] R. Alcotte et al., "Epitaxial growth of antiphase boundary free GaAs layer on $300 \mathrm{~mm} \mathrm{Si(001)} \mathrm{substrate} \mathrm{by} \mathrm{metalorganic} \mathrm{chemical} \mathrm{vapour}$ deposition with high mobility," APL Materials, vol. 4, no. 4, p. 046101, 2016.

[31] M. Tang et al., "Optimizations of Defect Filter Layers for 1.3- $\mu \mathrm{m}$ InAs/GaAs Quantum-Dot Lasers Monolithically Grown on Si Substrates," IEEE Journal of Selected Topics in Quantum Electronics, vol. 22, no. 6, pp. 50-56, 2016.

[32] H. Y. Liu et al., "Improved performance of $1.3 \mu \mathrm{m}$ multilayer InAs quantum-dot lasers using a high-growth-temperature GaAs spacer layer," Applied Physics Letters, vol. 85, no. 5, pp. 704-706, 2004.

[33] P. B. Deotare, M. W. McCutcheon, I. W. Frank, M. Khan, and M. Lončar, "High quality factor photonic crystal nanobeam cavities," Applied Physics Letters, vol. 94, no. 12, p. 121106, 2009.

[34] T.-W. Lu, L.-H. Chiu, P.-T. Lin, and P.-T. Lee, "One-dimensional photonic crystal nanobeam lasers on a flexible substrate," Applied Physics Letters, vol. 99, no. 7, p. 071101, 2011.

[35] S. G. Johnson and J. D. Joannopoulos, "Block-iterative frequencydomain methods for Maxwell's equations in a planewave basis," Optics express, vol. 8, no. 3, pp. 173-190, 2001.

[36] T. Zhou et al., "Microscale local strain gauges based on visible micro-disk lasers embedded in a flexible substrate," Optics Express, vol. 26, no. 13, pp. 16797-16804, 2018/06/25 2018.

[37] K.-Y. Jeong et al., "Electrically driven nanobeam laser," Nature communications, vol. 4, no. 1, pp. 1-6, 2013.

[38] M. Nomura et al., "Room temperature continuous-wave lasing in photonic crystal nanocavity," Optics Express, vol. 14, no. 13, pp. 6308-6315, 2006.

[39] K. Tanabe, M. Nomura, D. Guimard, S. Iwamoto, and Y. Arakawa, "Room temperature continuous wave operation of InAs/GaAs quantum dot photonic crystal nanocavity laser on silicon substrate," Optics Express, vol. 17, no. 9, pp. 7036-7042, 2009/04/27 2009.

[40] Y. Gong, B. Ellis, G. Shambat, T. Sarmiento, J. S. Harris, and J. Vučković, "Nanobeam photonic crystal cavity quantum dot laser," Optics express, vol. 18, no. 9, pp. 8781-8789, 2010. 